\title{
ON GOING-DOWN FOR SIMPLE OVERRINGS. III
}

\author{
DAVID E. DOBBS ${ }^{1}$ AND IRA J. PAPICK
}

\begin{abstract}
.
THEOREM 1. Let $R$ be an integral domain with quotient field $K$. The following three conditions are equivalent:

(a) $R \subset R[u]$ satisfies going-down $(G D)$ for each $u$ in $K$;

(b) $R \subset V$ satisfies $G D$ for each valuation overring $V$ of $R$;

(c) $R \subset S$ satisfies $G D$ for each domain $S$ containing $R$.

If (d) is the condition obtained by restricting the domains $S$ in (c) to be overrings of $R$, then (a) $\Leftrightarrow$ (d) has been proved in case $R$ is Krull or integrally closed finite-conductor (e.g., pseudo-Bézout) or Noetherian.

THEOREM 2. Let $R \subset T$ be domains such that either $\operatorname{Spec}(R)$ or $\operatorname{Spec}(T)$, as a poset under inclusion, is a tree. If $R \subset R[u, v]$ satisfies $G D$ for each $u$ and $v$ in $T$, then $R \subset T$ satisfies $G D$.
\end{abstract}

1. Introduction. Throughout this note, let $R$ be an integral domain with quotient field $K$. Our main purpose is to prove

THEOREM 1. The following three conditions on $R$ are equivalent:

(a) $R \subset R[u]$ satisfies going-down $(G D)$ for each $u$ in $K$.

(b) $R \subset V$ satisfies $G D$ for each valuation overring $V$ of $R$.

(c) $R \subset T$ satisfies $G D$ for each integral domain $T$ containing $R$.

If (d) is the condition on $R$ obtained by restricting the rings $T$ in condition (c) to be overrings of $R$, then the equivalence (a) $\Leftrightarrow$ (d) has already been established in case $R$ is: Krull [4, Corollary 10]; GCD (pseudo-Bézout) [3, Corollary 4.3]; more generally, integrally closed finite-conductor [4, Corollary 4]; or Noetherian [5, Corollary 2.3]. Further evidence suggesting the validity of (a) $\Leftrightarrow$ (d) was provided by: McAdam [12, Proposition 2], [10, Theorem 1], whose work yields the analogue of (a) $\Leftrightarrow$ (c) in which all the relevant extensions are assumed integral; and the first-named author [5, Proposition 3.5], who showed that (a) holds for each overring of $R$ if and only if the same is true of (d).

As in [5], if $R$ satisfies (d), we say that $R$ is a going-down ring (and write: $R$ is GD). Inasmuch as (c) $\Rightarrow$ (d) $\Rightarrow$ (b) trivially, Theorem 1 implies the equivalence of the four conditions. Thus, to use more terminology from [5], Theorem 1 shows: $R$ is $\mathrm{SGD} \Leftrightarrow R$ is GD. Examples of going-down rings are Prüfer domains, arbitrary integral domains of Krull dimension 1, and the rings constructed by iterated restrained power series in [5, Corollary 4.4].

Any unexplained terminology is standard, as in [6] and [8].

Received by the editors November 4, 1974.

AMS (MOS) subject classifications (1970). Primary 13F05, 13A15.

Key words and phrases. Going-down, treed domain, flat overring, valuation ring.

${ }^{1}$ Supported in part by NSF Grant GP-28409A \#2. 
2. Proofs. We start with a result which serves both to motivate Theorem 2 and to dispatch the difficult implication in Theorem 1. First, recall from [5] that a commutative ring $A$ is said to be treed in case $\operatorname{Spec}(A)$, as a poset under inclusion, is a tree, i.e. in case no maximal ideal of $A$ contains incomparable primes.

Proposition. Let $T$ be a quasilocal treed integral domain containing $R$. If $R \subset R[u]$ satisfies $G D$ for each $u$ in $T$, then $R \subset T$ satisfies $G D$.

Proof. Deny the result. By [8, Exercise 37 (i) $\Rightarrow$ (ii), p. 44], there exist $P$ in $\operatorname{Spec}(R)$ and $N$ in $\operatorname{Spec}(T)$ such that $N$ is minimal amongst primes of $T$ containing $P T$ and $N \cap R \neq P$. As $T$ is quasilocal treed, $N$ is the radical of $P T$. Thus, choosing $r$ in $(N \cap R) \backslash P$ leads to an equation $r^{m}=\sum p_{i} w_{i}$ for some $p_{i}$ in $P, w_{i}$ in $T$, and $m \geqslant 1$.

Now, the primes of $T$ are linearly ordered by inclusion and, by a result of Prekowitz [9, p. 29], we may relabel the $p_{j}$ so that, for each $i, p_{1}$ divides a power of $p_{i}$ (with quotient in $T$ ). Raising the above equation to a suitably high power, say the $t$ th, gives an element $w$ in $T$ such that $r^{m t}=p_{1} w$.

An application of [8, Theorem 10] produces $N_{1}$, minimal amongst primes of $R[w]$ contained in $N \cap R[w]$ and containing $P R[w]$. Since $R \subset R[w]$ satisfies GD, [8, Exercise 37 (ii) $\Rightarrow$ (i), p. 44] shows that $N_{1} \cap R=P$, whence $r$ is in $P$. This contradiction completes the proof.

Proof of Theorem 1. [5, Proposition 3.2] shows (b) $\Rightarrow$ (a), and (c) $\Rightarrow$ (b) trivially.

Finally, assume (a), let $T$ be an integral domain containing $R$, let $P \subset M$ be primes of $R$, and let $N$ be a prime of $T$ contracting to $M$. By [8, Theorem 56], $T$ is contained in a valuation domain $W$ whose maximal ideal contracts to $N$.

Let $V=W \cap K$. Then $V$ is a valuation ring (of $K$ ), hence Prüfer, so that $W$ is $V$-flat and, by [7, Corollaire 3.9.4(ii), p. 254], $V \subset W$ satisfies GD. However, since $V$ is quasilocal treed, the above Proposition shows that $R \subset V$ satisfies GD and, as GD is transitive, $R \subset W$ also satisfies GD. We thus obtain a prime $Q$ of $W$ contracting to $P$, so that $Q \cap T$ is contained in $N$ and also contracts to $P$. Thus, (a) $\Rightarrow$ (c), and the proof is complete.

The next result generalizes [5, Proposition 4.1(iii)].

Corollary. Let $V$ be a valuation domain of the form $F+M$, where $F$ is a field and $M$ is the maximal ideal of $V$. Let $T$ be a subring of $F$. Then $T$ is $G D$ if and only if $T+M$ is $G D$.

Proof. It will be convenient to use criterion (d) of the introduction in testing for going-down rings. By [1, Theorem 3.1], the overrings of $T+M$ are of two types: rings $S+M$ arising from $T$-subalgebras $S$ of $F$, and overrings of $V$. Since $V$ is GD, the now-familiar transitivity argument shows that $T+M$ is GD if and only if $T+M \subset S+M$ satisfies GD for each $T$-subalgebra $S$ of $F$. The description of primes in rings of the form $*+M[6$, Theorem $\mathrm{A}(\mathrm{c}),(\mathrm{d})$, (e), p. 560] readily implies that, for $S$ as above, $T+M \subset S+M$ satisfies GD if and only if $T \subset S$ satisfies GD. Thus, $T+M$ is GD if and only if $T \subset S$ satisfies GD for each $T \subset S \subset F$. By Theorem 1 (more precisely, since (d) $\Rightarrow$ (c) and $F$ contains all the overrings of $T$ ), the latter condition is equivalent to $T$ being GD, and the proof is complete. 
REMARK 1. We pause to note some earlier instances of the technicalities in the proof of the above Proposition. Apropos of Prekowitz' result, McAdam observed in line 4 of the proof of [11, Theorem 1] that any pair of radical ideals in a quasilocal treed domain are comparable. Our combinatorial argument reducing a high power of a sum in a quasilocal treed domain to a single term is put to other use in a proof of Wadsworth [9, p. 30].

The phrasing of the Proposition places that result in a growing class of statements (cf. [12, Proposition 2] and [10, Theorem 1], mentioned earlier) of the kind: " $R \subset T$ satisfies GD, if $R \subset S$ satisfies GD for certain $R$-algebras $S$ of finite type ". A result of this kind is given next for arbitrary treed $R$ or $T$.

THEOREM 2. Let $T$ be an integral domain containing $R$ such that either $R$ or $T$ is treed. If $R \subset R[u, v]$ satisfies $G D$ for each pair of elements $u, v$ in $T$, then $R \subset T$ satisfies $G D$.

Proof. Deny the result. The characterization of "going-down to $P$ " in $[8$, Exercise 37(iii), p. 44] then supplies a prime $P$ of $R$ and $Q$, minimal amongst primes of $T$ containing $P T$, such that $P T \cap(R \backslash P)(T \backslash Q) \neq \varnothing$. This produces an equation $\sum p_{i} w_{i}=r v$ for some $p_{i}$ in $P, w_{i}$ in $T, r$ in $R \backslash P$, and $v$ in $T \backslash Q$.

Suppose $R$ is treed. Then the primes of $R_{P}$ are linearly ordered by inclusion, so that the above-cited result of Prekowitz permits a relabelling such that $p_{1}$ divides powers of each $p_{i}$, with quotients in $R_{P}$. Taking a sufficiently high power (the $t$ th) of the above equation has as upshot an equation $p_{1} w z^{-1}$ $=r^{t} v^{t}$ for some $w$ in $T$ and $z$ in $R \backslash P$. We thus have $p w=b u$, with $p=p_{1}$ in $P, w$ in $T, b=r^{t} z$ in $R \backslash P$, and $u=v^{t}$ in $T \backslash Q$. If $T$ is treed, we argue similarly, obtaining quotients in $T_{Q}$ instead of $R_{P}$, and again find an equation of the form $p w=b u$.

After lowering $Q \cap R[w, u]$ to a prime minimal over $P R[w, u]$, this equation violates the characterization in [8, Exercise 37(iii), p. 44] of going-down (to $P$ ) for $R \subset R[w, u]$, which completes the proof.

REMARK 2. If $\mathcal{P}$ is a property which may be satisfied by extensions of commutative rings, let $\left(a_{\mathscr{P}}\right), \ldots,\left(\mathrm{d}_{\mathscr{P}}\right)$ be the statements obtained from $(\mathrm{a}), \ldots$, (d) in the introduction by replacing "GD" with "థ९⿱". Thus, Theorem 1 asserts the equivalence of $\left(\mathrm{a}_{\mathcal{P}}\right), \ldots,\left(\mathrm{d}_{\mathscr{P}}\right)$ in case $\mathscr{P}=\mathrm{GD}$. The corresponding equivalences for $\mathscr{P}^{\prime}=$ flat also hold (cf. [5, Proposition 3.1]), and characterize Prüfer domains. Let $\theta$ be given by: $A \theta B$ if and only if $\operatorname{Spec}(B) \rightarrow \operatorname{Spec}(A)$ is open. Since $\mathcal{P}^{\prime} \Rightarrow$ GD and $\theta \Rightarrow$ GD [7, Corollaire 3.9.4(ii) and (i), p. 254], it is worth noting that $\left(\mathrm{a}_{\theta}\right) \nRightarrow\left(\mathrm{d}_{\theta}\right)$. Indeed, as shown in [13], it follows easily from [14, Corollaire 2, p. 42] that any Prüfer $R$ satisfies $\left(\mathrm{a}_{\theta}\right)$, while compactness of Spec $(R)$ [2, Proposition 12 , p. 128] shows that any $R$ satisfying $\left(\mathrm{d}_{\theta}\right)$ must be quasisemilocal. Proofs of the equivalences $\left(\mathrm{b}_{\theta}\right) \Leftrightarrow\left(\mathrm{c}_{\theta}\right) \Leftrightarrow\left(\mathrm{d}_{\theta}\right)$ and a characterization of rings satisfying $\left(\mathrm{d}_{\theta}\right)$ will appear in [13].

Acknowledgment. The authors are grateful to $\mathrm{S}$. McAdam for his comments in regard to a preliminary draft of this paper.

\section{REFERENCES}

1. E. Bastida and R. Gilmer, Overrings and divisorial ideals of rings of the form $D+M$, Michigan Math. J. 20 (1973), 79-95. MR 48 \#2138.

2. N. Bourbaki, Eléments de mathématique. Fasc. XXVII. Algèbre commutative. Chaps. 1, 2, 
Actualités Sci. Indust., no. 1290, Hermann, Paris, 1961. MR 36\# 146.

3. J. Dawson and D. E. Dobbs, On going down in polynomial rings, Canad. J. Math. 26 (1974), 177--184.

4. D. E. Dobbs, On going down for simple overrings, Proc. Amer. Math. Soc. 39 (1973), 515519.

5. - On going down for simple overrings. II, Comm. Algebra 1 (1974), 439-458.

6. R. W. Gilmer, Jr., Multiplicative ideal theory, Queen's Papers in Pure and Appl. Math., no. 12, Queen's University, Kingston, Ont., 1968. MR 37 \#198.

7. A. Grothendieck and J. A. Dieudonné, Eléments de géométrie algébrique. I, Springer-Verlag, Berlin and New York, 1971.

8. I. Kaplansky, Commutative rings, Allyn and Bacon, Boston, Mass., 1970. MR 40\#7234.

9. I.- Topics in commutative ring theory. III, University of Chicago, Chicago, Ill. (mimeographed notes).

10. S. McAdam, Going down, Duke Math. J. 39 (1972), 633-636. MR 47 \#220.

11. Two conductor theorems, J. Algebra 23 (1972), 239-240. MR 46 \#306.

12. - Going down and open extensions, Canad. J. Math. (to appear).

13. I. J. Papick, Ph.D. Dissertation, Rutgers University, 1975.

14. M. Raynaud, Anneaux locaux henséliens, Lecture Notes in Math., vol. 169, SpringerVerlag, Berlin and New York, 1970. MR 43 \#252.

Department of Mathematics, Rutgers University, New Brunswick, New Jersey 08903

Current address: (D. E. Dobbs): Department of Mathematics, University of Tennessee, Knoxville, Tennessee 37916

Current address: (I. J. Papick): Department of Mathematics, Adelphi University, Garden City, Long Island, New York 11530 